Article

\title{
Some Fixed-Point Theorems in $b$-Dislocated Metric Space and Applications
}

\author{
Panda Sumati Kumari ${ }^{1, *(1)}$, Obaid Alqahtani ${ }^{2}$ and Erdal Karapınar ${ }^{3,4}$ \\ 1 Department of Mathematics, Basic Sciences and Humanities, GMR Institute of Technology, \\ Rajam 532127, India \\ 2 Department of Mathematics, King Saud University, Riyadh 11451, Saudi Arabia; obalgahtani@ksu.edu.sa \\ 3 Department of Mathematics, Atilim University, İncek, 06830 Ankara, Turkey; erdalkarapinar@yahoo.com \\ 4 Department of Medical Research, China Medical University, Taichung 40402, Taiwan \\ * Correspondence: mumy143143143@gmail.com or sumatikumari.p@gmrit.org
}

Received: 9 November 2018; Accepted: 26 November 2018; Published: 2 December 2018

check for updates

\begin{abstract}
In this article, we prove some fixed-point theorems in $b$-dislocated metric space. Thereafter, we propose a simple and efficient solution for a non-linear integral equation and non-linear fractional differential equations of Caputo type by using the technique of fixed point.
\end{abstract}

Keywords: fixed point; $b$-dislocated metric space (simply $b$-dislocated metric space); $F$-contraction; non-linear fractional differential equations of Caputo type

AMS Subject Classification (2000): 34A12; 45D05; 47H10; 47H30; 54H25

\section{Introduction}

The F-contraction is widely renowned, and has provided research contributions on various aspects in fixed-point theory for over 10 years.

Definition 1 ([1]). Let $(X, d)$ be a metric space. A mapping $T: X \rightarrow X$ is said to be a F-contraction on $(X, d)$ if there exist $F$ along with $C_{1}, C_{2}, C_{3}$ and $\tau>0$ such that

$$
d(T x, T y)>0 \Rightarrow \tau+F(d(T x, T y)) \leq F(d(x, y))
$$

From above we have:

- $\quad$ every F-contraction is necessarily continuous;

- $\quad$ every F-contraction is need not be a Banach contraction.

Now we present a list of certain F-contractive conditions for a self-map on a metric space:

(E1) $\quad \frac{1}{2} d(x, T x)<d(x, y) \Rightarrow \tau+F(d(T x, T y)) \leq F(d(x, y))$,

(E2) $\frac{1}{2} d(x, T x)<d(x, y) \Rightarrow \tau+F(d(T x, T y)) \leq F\left(d(x, y), d(x, T x), d(y, T y), \frac{d(x, T x)+d(y, T y)}{4}\right)$,

(E3) $\quad d(T x, T y)>0 \Rightarrow \tau+F(d(T x, T y)) \leq F\left\{\max \left(d(x, y), d(x, T x), d(y, T y), \frac{d(x, T y)+d(y, T x)}{2}\right)\right\}$

(E4) $\quad d(T x, T y)>0 \Rightarrow \tau+F(d(T x, T y)) \leq F(d(x, y)+L d(y, T x))$ and $d(T x, T y)>0 \Rightarrow \tau+F(d(T x, T y)) \leq F(d(x, y)+L d(x, T y))$

(E5) $\quad \omega(d(x, y))+F(d(T x, T y)) \leq F\left(f_{1} d(x, y)+f_{2} d(x, T x)+f_{3} d(y, T y)+f_{4} d(x, T y)+f_{5} d(y, T x)\right)$ 
(E6)

$$
\mathfrak{\omega}(d(x, y))+F(d(T x, T y)) \leq F(d(x, y))
$$

for all $x, y \in X$, where $\tau>0, T: X \rightarrow X, F: \mathbb{R}_{0}^{+} \rightarrow \mathbb{R}$ and $\omega:(0, \infty) \rightarrow(0, \infty)$.

Below is the list of necessary conditions which helps authors to establish the statements of the certain $F$-contractions and treated as main tool to obtain fixed points:

$\left(C_{1}\right) . F$ is strictly increasing;

$\left(C_{2}\right) . \lim _{n \rightarrow \infty} t_{1_{n}}=0$ if and only if $\lim _{n \rightarrow \infty} F\left(t_{1_{n}}\right)=-\infty$;

$\left(C_{3}\right)$. There exists $k \in(0,1)$ such that $\lim _{t_{1} \rightarrow 0^{+}} t_{1}^{k} F\left(t_{1}\right)=0$;

$\left(C_{4}\right)$. inf $F=-\infty$;

$\left(C_{5}\right) . F$ is continuous on $(0, \infty)$;

$\left(C_{6}\right) . \liminf _{u \rightarrow v^{+}} \mathscr{\omega}(u)>0$ for all $v \geq 0$;

$\left(C_{7}\right) . \lim _{v \rightarrow 0^{+}} F(v)=-\infty$.

We present a list of $F$-contractions and we named these conditions with their respective authors:

$1^{\circ}$. Secelean type $F$-contraction [2] if $T$ satisfies $(\star)$ along with $\left(C_{1}\right),\left(C_{4}\right)$ and $\left(C_{5}\right)$;

$2^{\circ}$. Piri type $F$-contraction [3] if $T$ satisfies $(E 1)$ along with $\left(C_{1}\right),\left(C_{4}\right)$ and $\left(C_{5}\right)$;

$3^{\circ}$. Karapinar type $F$-contraction [4] if $T$ satisfies (E2) along with $\left(C_{1}\right),\left(C_{4}\right)$ and $\left(C_{5}\right)$;

$4^{\circ}$. Wardowski-I type $F$-contraction [5] if $T$ satisfies $(E 3)$ along with $\left(C_{1}\right),\left(C_{2}\right)$ and $\left(C_{3}\right)$;

$5^{\circ}$. Minak type $F$-contraction [6] if $T$ satisfies $(E 3)$ along with $\left(C_{1}\right),\left(C_{2}\right)$ and $\left(C_{3}\right)$;

$6^{\circ}$. Minak-I type $F$-contraction [6] if $T$ satisfies $(E 4)$ along with $\left(C_{1}\right),\left(C_{2}\right)$ and $\left(C_{3}\right)$;

$7^{\circ}$. Vetro type $F$-contraction [7] if $T$ satisfies (E5) along with $\left(C_{1}\right),\left(C_{2}\right),\left(C_{3}\right)$ and $\left(C_{6}\right)$;

$8^{\circ}$. Wardowski-II type $F$-contraction [8] if $T$ satisfies $(E 6)$ along with $\left(C_{1}\right),\left(C_{6}\right)$ and $\left(C_{7}\right)$;

$9^{\circ}$. A. Lukacs and S. Kajanto type $F$-contraction [9] if $T$ satisfies $(\star)$ along with $\left(C_{1}\right)$ and $\left(C_{3}\right)$;

If we consider the $F$-contraction in the theory of fixed point, we can see various developments in it. More particularly, the techniques used to obtain fixed points in [1] have attracted several authors. In this scenario, many authors imposed various restrictions to obtain the existence of a fixed point (see for example [10-15]).

On the other hand, there are many generalizations on the concept of metric spaces in the literature. In particular, Matthews [16] introduced the notion of dislocated metric space under the name 'metric domains'. Later, in 2000, Hitzler and Seda [17] renamed these spaces as 'dislocated metric spaces' as below:

Definition 2 ([17]). A dislocated metric on a nonempty set $X$ is a function $d: X \times X \rightarrow[0,+\infty)$ such that for all $x, y, z \in X$. Then the following conditions hold good:

1. $d(x, y)=0 \Rightarrow x=y$;

2. $\quad d(x, y)=d(y, x)$

3. $d(x, y) \leq d(x, z)+d(z, y)$.

The pair $(X, d)$ is called a dislocated metric space.

Very recently, Alghamdi et al. [18] introduced b-dislocated metric space as below.

Definition 3 ([18]). A b-dislocated metric on a nonempty set $X$ is a function $d: X \times X \rightarrow[0,+\infty)$ such that for all $x, y, z \in X$ and $a$ constant $b \geq 1$ the following conditions hold good:

1. $d(x, y)=0 \Rightarrow x=y$;

2. $\quad d(x, y)=d(y, x)$;

3. $d(x, y) \leq k[d(x, z)+d(z, y)]$. 
The pair $(X, d)$ is called a $b$-dislocated metric space. Moreover, if $k=1$ then $b$-dislocated metric space becomes dislocated metric space as in $[16,17]$.

The structure properties of convergent, Cauchy and complete of $b$-dislocated metric space can be found in [18] as below:

Definition 4 ([18]). Let $(X, d)$ be a b-dislocated metric space, and let $\left\{x_{n}\right\}$ be a sequence of points of $X$. Then $\left\{x_{n}\right\}$ called,

- Convergent to $x$ if and only if $\lim _{n \rightarrow+\infty} d\left(x, x_{n}\right)=0$

- Cauchy if and only if $\lim _{m, n \rightarrow \infty} d\left(x_{n}, x_{m}\right)$ exists and is tends to finite.

- Complete if and only if every Cauchy sequence $\left\{x_{n}\right\}$ in $X$ converges to $x \in X$ this gives

$$
\lim _{m, n \rightarrow \infty} d\left(x_{n}, x_{m}\right)=0=\lim _{n \rightarrow \infty} d\left(x_{n}, x\right) .
$$

The study of dislocated metric space and its generalizations was very interesting and expanded very extensively. For more info, the reader can refer to [19-26].

Motivated by Wardowski [1], we introduce the notions of an extended F-contraction and weak-generalized $F$-contraction. Furthermore, we establish some fixed-point results for given named contractions. Thereafter, we propose a simple and efficient solution for a non-linear integral equation by using the technique of fixed point in the setting of $b$-dislocated metric space.

\section{Extended F-Contraction}

Now we start this section by introducing below definition.

Definition 5. Let $(X, d)$ be a b-dislocated metric space. A mapping $T: X \rightarrow X$ is said to be an Extended $F$-contraction if there exists $F \in \mathcal{F}$ and $\tau>0$ such that for all $x, y \in X$ with $d(T x, T y)>0$,

$$
\frac{1}{2 k} d(x, T x)<d(x, y) \Rightarrow \tau+F(d(T x, T y)) \leq F(\mathcal{A}(x, y))
$$

where,

$$
\begin{aligned}
\mathcal{A}(x, y)=\frac{\mathcal{A}_{1}(k+1)}{k} d(x, y)+\mathcal{A}_{2}[d(x, T x) & +d(y, T y)]+\mathcal{A}_{3}\left[\frac{d(x, T y)+d(y, T x)}{3 k}\right] \\
& +\mathcal{A}_{4}\left[\frac{d(x, x)+d(y, y)}{4 k}\right]
\end{aligned}
$$

here $\mathcal{A}_{1}, \mathcal{A}_{2}, \mathcal{A}_{3}, \mathcal{A}_{4} \geq 0, \frac{\mathcal{A}_{1}(k+1)}{k}+2\left(\mathcal{A}_{2}+\mathcal{A}_{3}+\mathcal{A}_{4}\right)<1$ and $F: \mathbb{R}^{+} \rightarrow \mathbb{R}$ is a mapping satisfying the following conditions:

1. $F$ is an order embedding. i.e., for all $t_{1}, t_{2} \in \mathbb{R}^{+}$we have $t_{1} \leq t_{2} \Rightarrow F\left(t_{1}\right) \leq F\left(t_{2}\right)$.

2. F is sub-additive, i.e., for $t_{1}, t_{2}, s_{1}, s_{2} \in \mathbb{R}^{+}$we have

$$
F\left(s_{1} t_{1}+s_{2} t_{2}\right) \leq s_{1} F\left(t_{1}\right)+s_{2} F\left(t_{2}\right)
$$

3. For every sequence $\left\{t_{1_{n}}\right\}_{n \in \mathbb{N}}$ of positive numbers

$$
\lim _{n \rightarrow \infty} t_{1_{n}}=0 \text { if and only if } \lim _{n \rightarrow \infty} F\left(t_{1_{n}}\right)=-\infty ;
$$

Let $\mathcal{F}$ denote the family of all functions $F: \mathbb{R}^{+} \rightarrow \mathbb{R}$ which satisfy conditions (1), (2) and (3). 
Under this new scenario, we will prove below theorem.

Theorem 1. Let $(X, d)$ be a complete $b$-dislocated metric space such that $d$ is a continuous function. If $T$ is an Extended F-contraction then $T$ has a unique fixed point.

Proof. Now, take $x \in X$ and build a sequence $\left\{x_{n}\right\}$ as follows:

$$
x_{n}=T x_{n-1}=T^{n} x, \text { for all } n \in \mathbb{N} \text { where } x_{0}=x .
$$

If there exists $n_{\star} \in \mathbb{N}$ such that $d\left(x_{n_{\star}}, T x_{n_{\star}}\right)=0$ then $x_{\star}=x_{n_{\star}}$ turn into a fixed point which completes the proof. As a result, we assume for every $n \in \mathbb{N}$,

$$
0<d\left(x_{n}, T x_{n}\right) .
$$

Therefore, from (1), we have

$$
\frac{1}{2 k} d\left(x_{n}, T x_{n}\right)<d\left(x_{n}, T x_{n}\right)=d\left(x_{n}, x_{n+1}\right), \text { for all } n \in \mathbb{N}
$$

Since $T$ is Extended $F$-contraction,

$$
\begin{aligned}
& \tau+F\left(d\left(T x_{n}, T x_{n+1}\right)\right) \leq F\left(\mathcal{A}\left(x_{n}, x_{n+1}\right)\right) \\
& \leq F\left\{\frac{\mathcal{A}_{1}(k+1)}{k} d\left(x_{n}, x_{n+1}\right)+\mathcal{A}_{2}\left[d\left(x_{n}, T x_{n}\right)+d\left(x_{n+1}, T x_{n+1}\right)\right]\right. \\
&\left.\quad+\mathcal{A}_{3}\left[\frac{d\left(x_{n}, T x_{n+1}\right)+d\left(x_{n+1}, T x_{n}\right)}{3 k}\right]+\mathcal{A}_{4}\left[\frac{d\left(x_{n}, x_{n}\right)+d\left(x_{n+1}, x_{n+1}\right)}{4 k}\right]\right\} \\
& \leq F\left\{\frac{\mathcal{A}_{1}(k+1)}{k} d\left(x_{n}, x_{n+1}\right)+\mathcal{A}_{2}\left[d\left(x_{n}, x_{n+1}\right)+d\left(x_{n+1}, x_{n+2}\right)\right]\right. \\
&\left.\quad+\mathcal{A}_{3}\left[\frac{d\left(x_{n}, x_{n+2}\right)+d\left(x_{n+1}, x_{n+1}\right)}{3 k}\right]+\mathcal{A}_{4}\left[\frac{d\left(x_{n}, x_{n}\right)+d\left(x_{n+1}, x_{n+1}\right)}{4 k}\right]\right\}
\end{aligned}
$$

From triangle inequality, we have,

$$
d\left(x_{n}, x_{n+2}\right) \leq k\left[d\left(x_{n}, x_{n+1}\right)+d\left(x_{n+1}, x_{n+2}\right)\right]
$$

and

$$
\begin{aligned}
d\left(x_{n+1}, x_{n+1}\right) & \leq 2 k d\left(x_{n+2}, x_{n+1}\right) \\
& \leq 2 k\left[d\left(x_{n+1}, x_{n+2}\right)+d\left(x_{n}, x_{n+1}\right)\right]
\end{aligned}
$$

By using (4) and (5),

$$
\frac{d\left(x_{n}, x_{n+2}\right)+d\left(x_{n+1}, x_{n+1}\right)}{3 k} \leq d\left(x_{n}, x_{n+1}\right)+d\left(x_{n+1}, x_{n+2}\right) .
$$

Also,

$$
\begin{aligned}
d\left(x_{n}, x_{n}\right) & \leq 2 k d\left(x_{n}, x_{n+1}\right) \\
& \leq 2 k\left[d\left(x_{n}, x_{n+1}\right)+d\left(x_{n+1}, x_{n+2}\right)\right]
\end{aligned}
$$

From (5) and (7),

$$
\frac{d\left(x_{n}, x_{n}\right)+d\left(x_{n+1}, x_{n+1}\right)}{4 k} \leq d\left(x_{n}, x_{n+1}\right)+d\left(x_{n+1}, x_{n+2}\right) .
$$


From (3), (7) and (8) we get

$$
\begin{aligned}
\tau+F\left(d\left(T x_{n}, T x_{n+1}\right)\right) \leq F\left\{\frac{\mathcal{A}_{1}(k+1)}{k} d\left(x_{n}, x_{n+1}\right)+\mathcal{A}_{2}\left[d\left(x_{n}, x_{n+1}\right)+d\left(x_{n+1}, x_{n+2}\right)\right]\right. \\
+\mathcal{A}_{3}\left[d\left(x_{n}, x_{n+1}\right)+d\left(x_{n+1}, x_{n+2}\right)\right] \\
\left.+\mathcal{A}_{4}\left[d\left(x_{n}, x_{n+1}\right)+d\left(x_{n+1}, x_{n+2}\right)\right]\right\}
\end{aligned}
$$

Since $F \in \mathcal{F}$,

$$
\begin{gathered}
\begin{array}{r}
\tau+F\left(d\left(x_{n+1}, x_{n+2}\right)\right) \leq \frac{\mathcal{A}_{1}(k+1)}{k} F\left(d\left(x_{n}, x_{n+1}\right)\right)+\mathcal{A}_{2} F\left(d\left(x_{n}, x_{n+1}\right)\right)+\mathcal{A}_{2} F\left(d\left(x_{n+1}, x_{n+2}\right)\right) \\
+\mathcal{A}_{3} F\left(d\left(x_{n}, x_{n+1}\right)\right)+\mathcal{A}_{3} F\left(d\left(x_{n+1}, x_{n+2}\right)\right) \\
+\mathcal{A}_{4} F\left(d\left(x_{n}, x_{n+1}\right)\right)+\mathcal{A}_{4} F\left(d\left(x_{n+1}, x_{n+2}\right)\right)
\end{array} \\
\leq\left[\frac{\mathcal{A}_{1}(k+1)}{k}+\mathcal{A}_{2}+\mathcal{A}_{3}+\mathcal{A}_{4}\right] F\left(d\left(x_{n}, x_{n+1}\right)\right)+\left(\mathcal{A}_{2}+\mathcal{A}_{3}+\mathcal{A}_{4}\right) F\left(d\left(x_{n+1}, x_{n+2}\right)\right)
\end{gathered}
$$

If we continue same scenario, we get,

$$
\begin{aligned}
F\left(d\left(x_{n}, T x_{n}\right)\right) & =F\left(d\left(x_{n}, x_{n+1}\right)\right) \\
& \leq F\left(d\left(x_{n-1}, x_{n}\right)\right)-\frac{\tau}{1-\left(\mathcal{A}_{2}+\mathcal{A}_{3}+\mathcal{A}_{4}\right)} \\
& \leq F\left(d\left(x_{n-2}, x_{n-1}\right)\right)-\frac{2 \tau}{1-\left(\mathcal{A}_{2}+\mathcal{A}_{3}+\mathcal{A}_{4}\right)} \\
& \leq F\left(d\left(x_{n-3}, x_{n-2}\right)\right)-\frac{3 \tau}{1-\left(\mathcal{A}_{2}+\mathcal{A}_{3}+\mathcal{A}_{4}\right)} \\
& \vdots \\
& \leq F\left(d\left(x_{0}, x_{1}\right)\right)-n \tau \\
& \leq F\left(d\left(x_{0}, T x_{0}\right)\right)-n \tau
\end{aligned}
$$

From (13), we obtain,

$$
\lim _{n \rightarrow \infty} F\left(d\left(x_{n}, T x_{n}\right)\right)=-\infty
$$


From (15) and from the hypothesis, we have

$$
\lim _{n \rightarrow \infty} F\left(d\left(x_{n}, T x_{n}\right)\right)=\lim _{n \rightarrow \infty} F\left(d\left(x_{n}, x_{n+1}\right)\right)=0 .
$$

Now, we claim that

$$
\lim _{n \rightarrow \infty} F\left(d\left(x_{n}, x_{m}\right)\right)=0 .
$$

i.e., $\left\{x_{n}\right\}$ is a Cauchy sequence. Let us suppose that $\left\{x_{n}\right\}$ is not a Cauchy sequence, then there exist $\epsilon>0$ and sequence $\{a(n)\}_{n=1}^{\infty}$ and $\{b(n)\}_{n=1}^{\infty}$ of natural numbers such that $a(n)>b(n)>n$, $d\left(x_{a(n)}, x_{b(n)}\right) \geq \epsilon$,

$$
d\left(x_{a(n)-1}, x_{b(n)}\right)<\frac{\epsilon}{k}, \text { for all } n \in \mathbb{N} .
$$

From the triangle inequality, we get,

$$
\begin{aligned}
\epsilon & \leq d\left(x_{a(n)}, x_{b(n)}\right) \leq k\left[d\left(x_{a(n)}, x_{a(n)-1}\right)+d\left(x_{a(n)-1}, x_{b(n)}\right)\right] \\
& \leq k d\left(x_{a(n)}, x_{a(n)-1}\right)+\epsilon \\
& =k d\left(x_{a(n)-1}, T x_{a(n)-1}\right)+\epsilon
\end{aligned}
$$

From (15) and (18) and the sandwich theorem, we get,

$$
\lim _{n \rightarrow \infty} d\left(x_{a(n)}, x_{b(n)}\right)=\epsilon, \text { for all } n \in \mathbb{N} .
$$

From Extended F-contraction, we have

$$
\begin{gathered}
\tau+F\left(d\left(T x_{a(n)}, T x_{b(n)}\right)\right) \leq F\left\{\frac{\mathcal{A}_{1}(k+1)}{k} d\left(x_{a(n)}, x_{b(n)}\right)+\mathcal{A}_{2}\left[d\left(x_{a(n)}, T x_{a(n)}\right)+d\left(x_{b(n)}, T x_{b(n)}\right)\right]\right. \\
+\mathcal{A}_{3}\left[\frac{d\left(x_{a(n)}, T x_{b(n)}\right)+d\left(x_{b(n)}, T x_{a(n)}\right)}{3 k}\right] \\
\left.+\mathcal{A}_{4}\left[\frac{d\left(x_{a(n)}, x_{a(n)}\right)+d\left(x_{b(n)}, x_{b(n)}\right)}{4 k}\right]\right\} \\
\leq F\left\{\frac{\mathcal{A}_{1}(k+1)}{k} d\left(x_{a(n)}, x_{b(n)}\right)+\mathcal{A}_{2}\left[d\left(x_{a(n)}, x_{a(n)+1}\right)+d\left(x_{b(n)}, x_{b(n)+1}\right)\right]\right. \\
+\mathcal{A}_{3}\left[\frac{d\left(x_{a(n)}, x_{b(n)+1}\right)+d\left(x_{b(n)}, x_{a(n)+1}\right)}{3 k}\right] \\
\left.+\mathcal{A}_{4}\left[\frac{d\left(x_{a(n)}, x_{a(n)}\right)+d\left(x_{b(n)}, x_{b(n)}\right)}{4 k}\right]\right\}
\end{gathered}
$$

Letting $n \rightarrow \infty$,

$$
\begin{aligned}
\tau+F(\epsilon) & \leq F\left[\frac{\mathcal{A}_{1}(k+1)}{k} \epsilon+\mathcal{A}_{3}\left(\frac{k(\epsilon)+k(\epsilon)}{3 k}\right)\right] \\
& \leq F\left(\frac{\mathcal{A}_{1}(k+1)}{k} \epsilon+\frac{2 \mathcal{A}_{3} \epsilon}{3}\right),
\end{aligned}
$$

which is a contradiction.

Hence $\lim _{m, n \rightarrow \infty} d\left(x_{n}, x_{m}\right)=0$. Thus, the sequence $\left\{x_{n}\right\}_{n=1}^{\infty}$ is a Cauchy in $X$.

Since $(X, d)$ is a Complete $b$-dislocated metric space, there exists $\omega \in X$ such that

$$
d(\omega, \omega)=\lim _{n \rightarrow \infty} d\left(x_{n}, \omega\right)=\lim _{m, n \rightarrow \infty} d\left(x_{n}, x_{m}\right)=0 .
$$


From (22)

$$
d\left(x_{n+1}, T \omega\right) \leq k\left[d\left(x_{n+1}, \omega\right)+d(\omega, T \omega)\right]
$$

letting $n \rightarrow \infty$,

$$
\lim _{n \rightarrow \infty} d\left(x_{n+1}, T \omega\right)=k b_{d}(\omega, T \omega)
$$

We now prove that for every $n \in N$

$$
\begin{aligned}
\frac{1}{2 k} d\left(x_{n}, T x_{n}\right) & <d\left(x_{n}, \omega\right) \text { or } \\
\frac{1}{2 k} d\left(T x_{n}, T^{2} x_{n}\right) & <d\left(T x_{n}, \omega\right) \quad \forall n \in \mathbb{N}
\end{aligned}
$$

Arguing by contradiction, we assume that there exists $e \in \mathbb{N}$ such that

$$
\begin{aligned}
\frac{1}{2 k} d\left(x_{e}, T x_{e}\right) & \geq d\left(x_{e}, \omega\right) \text { and } \\
\frac{1}{2 k} d\left(T x_{e}, T^{2} x_{e}\right) & \geq d\left(T x_{e}, \omega\right)
\end{aligned}
$$

Now from (12),

$$
d\left(T x_{e}, T^{2} x_{e}\right)<d\left(x_{e}, T x_{e}\right)
$$

From (25) and (26),

$$
\begin{aligned}
d\left(x_{e}, T x_{e}\right) & \leq k\left[d\left(x_{e}, \omega\right)+d\left(\omega, T x_{e}\right)\right] \\
& \leq k\left[\frac{1}{2 k} d\left(x_{e}, T x_{e}\right)+\frac{1}{2 k} d\left(T x_{e}, T^{2} x_{e}\right)\right] \\
& <k\left[\frac{1}{2 k} d\left(x_{e}, T x_{e}\right)+\frac{1}{2 k} d\left(x_{e}, T x_{e}\right)\right] \\
& =d\left(x_{e}, T x_{e}\right),
\end{aligned}
$$

which is a contradiction. Hence (24) holds.

Suppose $\frac{1}{2 k} d\left(x_{n}, T x_{n}\right)<d\left(x_{n}, \omega\right)$ is satisfied and $d(\omega, T \omega)>0$. From (23) there exists $N_{1} \in \mathbb{N}$ such that $d\left(T x_{n}, T \omega\right)=d\left(x_{n+1}, T \omega\right)>0$ for $n \geq N_{1}$. Then from Extended $F$-contraction (with $n \geq N_{1}$ ), we have,

$$
\begin{gathered}
\tau+F\left(d\left(x_{n+1}, T \omega\right)\right)=\tau+F\left(d\left(T x_{n}, T \omega\right)\right) \\
\leq F\left\{\frac{\mathcal{A}_{1}(k+1)}{k} d\left(x_{n}, \omega\right)+\mathcal{A}_{2}\left[d\left(x_{n}, T x_{n}\right)+d(\omega, T \omega)\right]\right. \\
+\mathcal{A}_{3}\left[\frac{d\left(x_{n}, T \omega\right)+d\left(\omega, T x_{n}\right)}{3 k}\right] \\
\left.\quad+\mathcal{A}_{4}\left[\frac{d\left(x_{n}, x_{n}\right)+d(\omega, \omega)}{4 k}\right]\right\} \\
\leq F\left\{\frac{\mathcal{A}_{1}(k+1)}{k} d\left(x_{n}, \omega\right)+\mathcal{A}_{2}\left[d\left(x_{n}, x_{n+1}\right)+d(\omega, T \omega)\right]\right. \\
+\mathcal{A}_{3}\left[\frac{d\left(x_{n}, T \omega\right)+d\left(\omega, x_{n+1}\right)}{3 k}\right] \\
\left.+\mathcal{A}_{4}\left[\frac{d\left(x_{n}, x_{n}\right)+d(\omega, \omega)}{4 k}\right]\right\}
\end{gathered}
$$


From (23) there exists $N_{k} \in \mathbb{N}$ with $\left(N_{k} \geq N_{1}\right)$ such that for all $n \geq N_{k}$.

$$
\begin{gathered}
\tau+F\left(d\left(x_{n+1}, T \omega\right)\right) \leq F\left\{\frac{\mathcal{A}_{1}(k+1)}{k} d\left(x_{n+1}, \omega\right)+\mathcal{A}_{2} d(\omega, T \omega)\right. \\
+\mathcal{A}_{3}\left[k \frac{d\left(x_{n+1}, T \omega\right)+d\left(\omega, x_{n+1}\right)}{3 k}\right] \\
\left.+\mathcal{A}_{4}\left[2 s \frac{d(\omega, T \omega)}{4 k}\right]\right\} \\
\leq F\left\{\mathcal{A}_{2}\left[d(\omega, T \omega)+\frac{\mathcal{A}_{3}}{3} d\left(x_{n+1}, T \omega\right)+\mathcal{A}_{4} \frac{d(\omega, T \omega)}{2}\right\},\right.
\end{gathered}
$$

which implies, $\left(1-\frac{\mathcal{A}_{3}}{3}\right) F\left(d\left(x_{n+1}, T \omega\right)\right) \leq\left(\mathcal{A}_{3}+\frac{\mathcal{A}_{4}}{2}\right) F(d(\omega, T \omega))-\tau$

Letting $n \rightarrow \infty$,

$$
\begin{aligned}
F(d(\omega, T \omega)) & \leq \frac{\mathcal{A}_{2}+\frac{\mathcal{A}_{4}}{2}}{1-\frac{\mathcal{A}_{3}}{3}} F(d(\omega, T \omega))-\frac{\tau}{1-\frac{\mathcal{A}_{3}}{3}} \\
& <\frac{\mathcal{A}_{2}+\frac{\mathcal{A}_{4}}{2}}{1-\frac{\mathcal{A}_{3}}{3}} F(d(\omega, T \omega)) \\
& <F(d(\omega, T \omega)) \forall n \geq N_{k}
\end{aligned}
$$

which is a contradiction.

Now suppose $\frac{1}{2 k} d\left(T x_{n}, T^{2} x_{n}\right)<d\left(T x_{n}, \omega\right)$ of (24) is true with $d(\omega, T \omega)>0$. Noted from (23), there exists $N_{p} \in \mathbb{N}$ such that $d\left(T\left(T x_{n}\right), T \omega\right)=d\left(x_{n+2}, T \omega\right)>0$ for $n \geq N_{p}$. Then from our assumption with $n \geq N_{p}$, we have

$$
\begin{aligned}
& \tau+F\left(d\left(x_{n+2}, T \omega\right)\right)=+F\left(d\left(T^{2} x_{n}, T \omega\right)\right) \\
& \leq F\left\{\frac{\mathcal{A}_{1}(k+1)}{k} d\left(T x_{n}, \omega\right)+\mathcal{A}_{2}\left[d\left(T x_{n}, T^{2} x_{n}\right)+d(\omega, T \omega)\right]\right. \\
& \quad+\mathcal{A}_{3}\left[\frac{d\left(T x_{n}, T \omega\right)+d\left(\omega, T^{2} x_{n}\right)}{3 k}\right] \\
&\left.\quad+\mathcal{A}_{4}\left[\frac{d\left(T x_{n}, T x_{n}\right)+d(\omega, \omega)}{4 k}\right]\right\} \\
& \leq F\left\{\frac{\mathcal{A}_{1}(k+1)}{k} d\left(x_{n+1}, \omega\right)+\mathcal{A}_{2}\left[d\left(x_{n+1}, x_{n+2}\right)+d(\omega, T \omega)\right]\right. \\
& \quad+\mathcal{A}_{3}\left[\frac{d\left(x_{n+1}, T \omega\right)+d\left(\omega, x_{n+2}\right)}{3 k}\right] \\
&\left.\quad+\mathcal{A}_{4}\left[\frac{d\left(x_{n+1}, x_{n+1}\right)+d(\omega, \omega)}{4 k}\right]\right\} \\
& \leq F\left(\mathcal{A}_{2} d(\omega, T \omega)+\frac{\mathcal{A}_{3}}{3 k} k b_{d}(\omega, T \omega)\right) \\
& \leq \mathcal{A}_{2} F(d(\omega, T \omega))+\frac{\mathcal{A}_{3}}{3} F(d(\omega, T \omega))
\end{aligned}
$$

Letting $n \rightarrow \infty$,

$$
\tau+F\left(d(\omega, T \omega) \leq\left[\mathcal{A}_{2}+\frac{\mathcal{A}_{3}}{3}\right] F(d(\omega, T \omega)\right.
$$




$$
\begin{aligned}
F(d(\omega, T \omega) & \leq\left[\mathcal{A}_{2}+\frac{\mathcal{A}_{3}}{3}\right] F(d(\omega, T \omega))-\tau \\
& <\left[\mathcal{A}_{2}+\frac{\mathcal{A}_{3}}{3}\right] F(d(\omega, T \omega)) \\
& <F(d(\omega, T \omega))
\end{aligned}
$$

which is a contradiction.

Hence we conclude that $d(\omega, T \omega)=0$, which gives $\omega=T \omega$. Therefore, $\omega$ is a fixed point of $T$.

To prove uniqueness, let $\omega$ and $\omega^{*}$ are two fixed points of $T$ such that $\omega \neq \omega^{*}$, which means,

$$
\begin{aligned}
\frac{1}{2 k} d\left(\omega, T \omega^{*}\right) & <d\left(\omega, T \omega^{*}\right) \\
& =d\left(\omega, \omega^{*}\right)
\end{aligned}
$$

Thus, from, Extended F-contraction,

$$
\begin{aligned}
& \tau+F\left(d\left(T \omega, T \omega^{*}\right)\right) \leq F\left(\mathcal{A}\left(\omega, \omega^{*}\right)\right) \\
& \leq F\left\{\frac{\mathcal{A}_{1}(k+1)}{k} d\left(\omega, \omega^{*}\right)+\mathcal{A}_{2}\left[d(\omega, T \omega)+d\left(\omega^{*}, T \omega^{*}\right)\right]\right. \\
&\left.\quad+\mathcal{A}_{3}\left[\frac{d\left(\omega, T \omega^{*}\right)+d\left(\omega^{*}, T \omega\right)}{3 k}\right]+\mathcal{A}_{4}\left[\frac{d\left(\omega, \omega^{*}\right)+d\left(\omega^{*}, \omega^{*}\right)}{4 k}\right]\right\} \\
& \leq F\left\{\frac{\mathcal{A}_{1}(k+1)}{k} d\left(\omega, \omega^{*}\right)+\mathcal{A}_{2}\left[d(\omega, \omega)+d\left(\omega^{*}, \omega^{*}\right)\right]\right. \\
&\left.+\mathcal{A}_{3}\left[\frac{d\left(\omega, \omega^{*}\right)+d\left(\omega^{*}, \omega\right)}{3 k}\right]\right\}
\end{aligned}
$$

From (22), $d(\omega, \omega)=d\left(\omega^{*}, \omega^{*}\right)=0$. Therefore (34) becomes:

$$
\begin{aligned}
\tau+F\left(d\left(\omega, \omega^{*}\right)\right) & \leq F\left\{\frac{\mathcal{A}_{1}(k+1)}{k} d\left(\omega, \omega^{*}\right)+2 \mathcal{A}_{3} \frac{d\left(\omega, \omega^{*}\right)}{3 k}\right\} \\
& \leq\left(\frac{\mathcal{A}_{1}(k+1)}{k}+\frac{2 \mathcal{A}_{3}}{3 k}\right) F\left(d\left(\omega, \omega^{*}\right)\right) \\
& =\left(\frac{3 \mathcal{A}_{1}(k+1)+2 \mathcal{A}_{3} k}{k}\right) F\left(d\left(\omega, \omega^{*}\right)\right) \\
& <F\left(d\left(\omega, \omega^{*}\right)\right)
\end{aligned}
$$

Thus, $F\left(d\left(\omega, \omega^{*}\right)\right)<F\left(d\left(\omega, \omega^{*}\right)\right)-\tau$, this gives a contradiction. Hence $\omega=\omega^{*}$.

Example 1. Consider $X=\{0,1,2\}$. Let $d: X \times X \rightarrow[0, \infty)$ be a mapping defined by

$$
\begin{gathered}
d(0,0)=d(1,1)=0, d(2,2)=2.5 ; \\
d(0,2)=d(2,0)=2, d(1,2)=d(2,1)=3 ; \\
d(0,1)=d(1,0)=1.5 .
\end{gathered}
$$

We can easily prove that $(X, d)$ is a $b$-dislocated metric space with $k=2$. Clearly $(X, d)$ is a complete b-dislocated metric space.

Let $T: X \rightarrow X$ be given by $T 0=T 1=0$ and $T 2=1$. Suppose that $F(v)=\frac{-1}{v}+v$.

Case-1: Let $x=0$, now $d(T 0, T 0)=d(T 0, T 1)=d(0,0)=0$. 
So, we need to consider $y=2$. Then we have $0.25 d(0, T 0)<d(0,2)$.

Consider,

$$
\begin{aligned}
\mathcal{A}(0,2)= & \mathcal{A}_{1} 1.5 d(0,2)+\mathcal{A}_{2}[d(0, T 0)+d(2, T 2)]+\mathcal{A}_{3}\left[\frac{d(0, T 2)+d(2, T 0)}{6}\right] \\
& +\mathcal{A}_{4}\left[\frac{d(0,0)+d(2,2)}{8}\right] \\
= & \mathcal{A}_{1} 1.5 \times 2+\mathcal{A}_{2}[d(0,0)+d(2,1)]+\mathcal{A}_{3}\left[\frac{d(0,1)+d(2,0)}{6}\right] \\
& +\mathcal{A}_{4}\left[\frac{d(0,0)+d(2,2)}{8}\right] \\
= & 3 \mathcal{A}_{1}+\mathcal{A}_{2}[0+3]+\mathcal{A}_{3}\left[\frac{1.5+2}{6}\right]+\mathcal{A}_{4}\left[\frac{0+2.5}{8}\right] \\
= & 3 \mathcal{A}_{1}+3 \mathcal{A}_{2}+0.58 \mathcal{A}_{3}+0.31 \mathcal{A}_{4}
\end{aligned}
$$

Let $\mathcal{A}_{1}=0.56, \mathcal{A}_{2}=\mathcal{A}_{3}=\mathcal{A}_{4}=0$ such that $1.5 \mathcal{A}_{1}+2\left(\mathcal{A}_{2}+\mathcal{A}_{3}+\mathcal{A}_{4}\right)<1$.

Now, $\mathcal{A}(0,2)=1.68$.

Consider,

$$
\begin{aligned}
F(d(T 0, T 2))-F(\mathcal{A}(0,2)) & =F(d(0,1))-F(\mathcal{A}(0,2)) \\
& =F(1.5)-F(1.68) \\
& =-0.66+1.5+0.59-1.68 \\
& =-0.25 \\
& =-\tau
\end{aligned}
$$

In this case, for $\tau=0.25, T$ satisfies all the conditions of Theorem 1 and 0 is the unique fixed point.

Case-2: Let $x=1$, now $d(T 0, T 1)=d(T 1, T 1)=d(0,0)=0$. So, we must consider $y=2$.

$$
d(1, T 1)=d(1,0)=1.5, d(1,2)=3 .
$$

Therefore $\frac{1}{4} d(1, T 1)<d(1,2)$.

$$
\begin{aligned}
\mathcal{A}(1,2)= & \mathcal{A}_{1} 1.5 d(1,2)+\mathcal{A}_{2}[d(1, T 1)+d(2, T 2)]+\mathcal{A}_{3}\left[\frac{d(1, T 2)+d(2, T 1)}{6}\right] \\
& +\mathcal{A}_{4}\left[\frac{d(1,1)+d(2,2)}{8}\right] \\
= & \mathcal{A}_{1} 1.5 \times 3+\mathcal{A}_{2}[d(1,0)+d(2,1)]+\mathcal{A}_{3}\left[\frac{d(1,1)+d(2,0)}{6}\right] \\
& +\mathcal{A}_{4}\left[\frac{d(1,1)+d(2,2)}{8}\right] \\
= & 4.5 \mathcal{A}_{1}+\mathcal{A}_{2}[1.5+3]+\mathcal{A}_{3}\left[\frac{0+2}{6}\right]+\mathcal{A}_{4}\left[\frac{0+2.5}{8}\right] \\
= & 4.5 \mathcal{A}_{1}+4.5 \mathcal{A}_{2}+0.33 \mathcal{A}_{3}+0.31 \mathcal{A}_{4}
\end{aligned}
$$

Let $\mathcal{A}_{1}=0.56, \mathcal{A}_{2}=\mathcal{A}_{3}=\mathcal{A}_{4}=0$ such that $1.5 \mathcal{A}_{1}+2\left(\mathcal{A}_{2}+\mathcal{A}_{3}+\mathcal{A}_{4}\right)<1$. 
Consider,

$$
\begin{aligned}
F(d(T 1, T 2))-F(\mathcal{A}(1,2)) & =F(d(0,1))-F(\mathcal{A}(1,2)) \\
& =F(1.5)-F(2.52) \\
& =-1.29 \\
& =-\tau
\end{aligned}
$$

In this case, for $\tau=1.29, T$ satisfies all the conditions of Theorem 1 and 0 is the unique fixed point.

Case-3: Let $x=2$, now $d(T 2, T 2)=0$. So, we need only consider the case $y \in\{0,1\}$.

Subcase-I: If $y=0$. Please note that $0.25 d(2, T 2)<d(2,0)$ as $0.75=0.25 d(2,1)<d(2,0)=2$.

$$
\tau+F(d(T 2, T 0)) \leq F(\mathcal{A}(2,0))
$$

follows as in Case-1.

Subcase-II: If $y=1$, note that $0.25 d(2, T 2)<d(2,1)$ as $0.75=0.25 d(2,1)<d(2,1)=3$.

$$
\tau+F(d(T 2, T 1)) \leq F(\mathcal{A}(2,1)),
$$

follows as in Case-2.

Hence $T$ is an extended $F$-contraction and it is clear that 0 is the fixed point of $T$.

If we take $k=1$, then the above theorem reduces to below corollary.

Corollary 1. Let $(X, d)$ be a dislocated metric space such that $d$ is a continuous function. If $T$ is an extended $F$-contraction, i.e., there exists $F \in \mathcal{F}$ and $\tau>0$ such that for all $x, y \in X$ with $d(T x, T y)>0$,

$$
\frac{1}{2} d(x, T x)<d(x, y) \Rightarrow \tau+F(d(T x, T y)) \leq F(\mathcal{A}(x, y))
$$

where,

$$
\begin{gathered}
\mathcal{A}(x, y)=2 \mathcal{A}_{1} d(x, y)+\mathcal{A}_{2}[d(x, T x)+d(y, T y)]+\mathcal{A}_{3}\left[\frac{d(x, T y)+d(y, T x)}{3}\right] \\
+\mathcal{A}_{4}\left[\frac{d(x, x)+d(y, y)}{4}\right]
\end{gathered}
$$

here $\mathcal{A}_{1}, \mathcal{A}_{2}, \mathcal{A}_{3}, \mathcal{A}_{4} \geq 0,2 \mathcal{A}_{1}+2\left(\mathcal{A}_{2}+\mathcal{A}_{3}+\mathcal{A}_{4}\right)<1$. Then $T$ has a unique fixed point.

If we take $\mathcal{A}_{2}=\mathcal{A}_{3}=\mathcal{A}_{4}=0$ and $\mathcal{A}_{1}<\frac{1}{2}$ in above theorem, we get below corollary.

Corollary 2. Let $(X, d)$ be a b-dislocated metric space. If $T$ is an extended F-contraction, i.e., there exists $F \in \mathcal{F}$ and $\tau>0$ such that for all $x, y \in X$ with $d(T x, T y)>0, \frac{1}{2} d(x, T x)<d(x, y) \Rightarrow \tau+F(d(T x, T y)) \leq$ $F(\mathcal{A}(x, y))$, here, $\left.\mathcal{A}(x, y)=2 \mathcal{A}_{1} d(x, y)\right)$. Then $T$ has a unique fixed point.

\section{Weak-Generalized F-Contraction}

Definition 6. Let $(X, d)$ be a b-dislocated metric space and $T: X \rightarrow X$ be a mapping. Then $T$ is said to be a Weak-generalized F-contraction if $F$ along with $C_{1}, C_{2}, C_{3}$ and there exists $\tau>0$ such that $\forall x, y \in X$,

$$
d(T x, T y)>0 \Rightarrow \tau+F(d(T x, T y)) \leq F(\mathcal{S}(x, y))
$$


where

$$
\begin{array}{r}
\mathcal{S}(x, y)=\max \left\{d(x, y), d(x, T x), d(y, T y), \frac{d(x, T x)+d(y, T y)}{2},\right. \\
\frac{d(x, T x) d(y, T y)}{1+d(x, y)}, \frac{d(y, T y)[1+d(x, T x)]}{1+d(x, y)}, \\
\left.\frac{d^{2}(x, T x) d(y, T y)}{1+d^{2}(x, y)}\right\}
\end{array}
$$

Theorem 2. Let $(X, d)$ be a b-dislocated metric space such that $d$ is continuous functional. If $T$ is a Weak-generalized F-contraction, then $T$ has a unique fixed point.

Proof. Choose an arbitrary point $x_{0} \in X$ and define a sequence $\left\{x_{n}\right\}$ in $X$ by $x_{n}=T x_{n-1}$ for $n \in\{1,2,3 \ldots\}$.

i.e., $x_{1}=T x_{0}, x_{2}=T x_{1}=T\left(T x_{0}\right)=T^{2} x_{0}, \ldots x_{n}=T x_{n-1}=T^{n} x_{0}$. If $d\left(x_{n_{0}}, x_{n_{0}+1}\right)=0$ for some $n_{0} \in\{0,1,2 \ldots\}$, then $x_{n_{0}}=x_{n_{0}+1}$, which yields $x_{n_{0}}=T x_{n_{0}}$, and so $T$ has a fixed point. Then there is nothing left to prove, and our proof is complete.

Thus, let us assume $d\left(x_{n}, x_{n+1}\right)>0$ for every $n \in\{0,1,2 \ldots\}$. i.e., $d\left(T x_{n-1}, T x_{n}\right)>0$.

Now using (36), we get,

$$
\tau+F\left(k b_{d}\left(T x_{n-1}, T x_{n}\right)\right) \leq F\left(\mathcal{S}\left(x_{n-1}, x_{n}\right)\right)
$$

where

$$
\begin{aligned}
\mathcal{S}\left(x_{n-1}, x_{n}\right)= & \max \left\{d\left(x_{n-1}, x_{n}\right), d\left(x_{n-1}, T x_{n-1}\right), d\left(x_{n}, T x_{n}\right), \frac{d\left(x_{n-1}, T x_{n-1}\right)+d\left(x_{n}, T x_{n}\right)}{2},\right. \\
\frac{d\left(x_{n}, T x_{n}\right)\left[1+d\left(x_{n-1}, T x_{n-1}\right)\right]}{1+d\left(x_{n-1}, x_{n}\right)}, \frac{d\left(x_{n-1}, T x_{n-1}\right) d\left(x_{n}, T x_{n}\right)}{1+d\left(x_{n-1}, x_{n}\right)}, & \left.\frac{d^{2}\left(x_{n-1}, T x_{n-1}\right) d\left(x_{n}, T x_{n}\right)}{1+d^{2}\left(x_{n-1}, x_{n}\right)}\right\} \\
= & \max \left\{d\left(x_{n-1}, x_{n}\right), d\left(x_{n-1}, x_{n}\right), d\left(x_{n}, x_{n+1}\right), \frac{d\left(x_{n-1}, x_{n}\right)+d\left(x_{n}, x_{n+1}\right)}{2},\right. \\
\frac{d\left(x_{n}, x_{n+1}\right)\left[1+d\left(x_{n-1}, x_{n}\right)\right]}{1+d\left(x_{n-1}, x_{n}\right)}, \frac{d\left(x_{n-1}, x_{n}\right) d\left(x_{n+1}, x_{n}\right)}{1+d\left(x_{n-1}, x_{n}\right)}, & \left.\frac{d^{2}\left(x_{n-1}, x_{n}\right) d\left(x_{n}, x_{n+1}\right)}{1+d^{2}\left(x_{n-1}, x_{n}\right)}\right\} \\
& \leq \max \left\{d\left(x_{n-1}, x_{n}\right), d\left(x_{n}, x_{n+1}\right)\right\}
\end{aligned}
$$

This yields,

$$
\tau+F\left(k B_{d}\left(x_{n}, x_{n+1}\right)\right) \leq F\left(\max \left\{d\left(x_{n-1}, x_{n}\right), d\left(x_{n}, x_{n+1}\right)\right\}\right)
$$

If $\max \left\{d\left(x_{n-1}, x_{n}\right), d\left(x_{n}, x_{n+1}\right)\right\}=d\left(x_{n}, x_{n+1}\right)$ then $\tau+F\left(k b_{d}\left(x_{n}, x_{n+1}\right)\right) \leq F\left(d\left(x_{n}, x_{n+1}\right)\right)$, which is a contradiction, since $\tau>0$. 
Hence $\max \left\{d\left(x_{n-1}, x_{n}\right), d\left(x_{n}, x_{n+1}\right)\right\}=d\left(x_{n-1}, x_{n}\right)$. Thus, (40) reduces to $\tau+$ $F\left(k B_{d}\left(x_{n}, x_{n+1}\right)\right) \leq F\left(d\left(x_{n-1}, x_{n}\right)\right)$. Therefore, we obtain,

$$
\begin{aligned}
F\left(k^{n} d\left(x_{n}, x_{n+1}\right)\right) & \leq F\left(k^{n-1} d\left(x_{n-1}, x_{n}\right)\right)-\tau \\
& \leq F\left(k^{n-2} d\left(x_{n-2}, x_{n-1}\right)\right)-2 \tau \\
& \vdots \\
& \leq F\left(k^{0} d\left(x_{0}, x_{1}\right)\right)-n \tau
\end{aligned}
$$

Applying $\lim _{n \rightarrow \infty}$ in above, we get,

$$
\lim _{n \rightarrow \infty} F\left(k^{n} d\left(x_{n}, x_{n+1}\right)\right)=-\infty .
$$

Thus, by using $\left(F_{2}\right)$ of Wardowski, we get,

$$
\left.\lim _{n \rightarrow \infty} k^{n} d\left(x_{n}, x_{n+1}\right)\right)=0 .
$$

Thus, from $\left(F_{3}\right)$ there exists $\vartheta \in(0,1)$ such that

$$
\lim _{n \rightarrow \infty}\left[k^{n} d\left(x_{n}, x_{n+1}\right)\right]^{\vartheta} F\left(d\left(x_{n}, x_{n+1}\right)\right)=0 .
$$

By (41), the following holds good for all $n \in\{1,2,3 \ldots\}$,

$$
\begin{aligned}
{\left[k^{n} d\left(x_{n}, x_{n+1}\right)\right]^{\vartheta} F\left(d\left(x_{n}, x_{n+1}\right)\right)-\left[k^{n} d\left(x_{n}, x_{n+1}\right)\right]^{\vartheta} F\left(d\left(x_{0}, x_{1}\right)\right) } & \leq-\left[k^{n} d\left(x_{n}, x_{n+1}\right)\right]^{\vartheta} n \tau \\
& \leq 0
\end{aligned}
$$

Letting $n \rightarrow \infty$ in (42), we get

$$
\lim _{n \rightarrow \infty} n\left[k^{n} d\left(x_{n}, x_{n+1}\right)\right]^{\vartheta}=0 .
$$

From (43), there exists $N_{1} \in\{1,2,3 \ldots\}$ such that $n\left[d\left(x_{n}, x_{n+1}\right)\right]^{\vartheta} \leq 1$ for all $n \geq N_{1}$. Therefore, we have for all $n \geq N_{1}$.

$$
k^{n} d\left(x_{n}, x_{n+1}\right) \leq \frac{1}{n^{\frac{1}{\vartheta}}}
$$

Now to prove that $\left\{x_{n}\right\}$ is a Cauchy sequence, consider $m, n \in N$ such that $m>n \geq N_{1}$. Using the triangle inequality and from (44), we have,

$$
\begin{aligned}
d\left(x_{n}, x_{m}\right) & \leq k\left[d\left(x_{n}, x_{n+1}\right)+d\left(x_{n+1}, x_{m}\right)\right] \\
& \leq k d\left(x_{n}, x_{n+1}\right)+k\left[k\left(d\left(x_{n+1}, x_{n+2}\right)+d\left(x_{n+2}, x_{m}\right)\right)\right] \\
& \leq k b_{d}\left(x_{n}, x_{n+1}\right)+k^{2} d\left(x_{n+1}, x_{n+2}\right)+k^{2} d\left(x_{n+2}, x_{m}\right) \\
& \vdots \\
& \leq k b_{d}\left(x_{n}, x_{n+1}\right)+k^{2} d\left(x_{n+1}, x_{n+2}\right)+k^{3} d\left(x_{n+2}, x_{n+3}\right)+\ldots+k^{m-1} d\left(x_{m-1}, x_{m}\right)
\end{aligned}
$$


Thus,

$$
\begin{aligned}
d\left(x_{n}, x_{m}\right) & \leq \sum_{e=n}^{m-1} k^{e} d\left(x_{e}, x_{e+1}\right) \\
& \leq \sum_{e=n}^{\infty} k^{e} d\left(x_{e}, x_{e+1}\right) \\
& \leq \sum_{e=n}^{\infty} \frac{1}{e^{\frac{1}{\vartheta}}}
\end{aligned}
$$

which is convergent, passing to limit $n \rightarrow \infty$, then we get $\lim _{n \rightarrow \infty} d\left(x_{n}, x_{m}\right)=0$. This yields that $\left\{x_{n}\right\}$ is a Cauchy sequence in $(X, d)$.

Since $(X, d)$ is a complete $b$-dislocated metric space, the sequence $\left\{x_{n}\right\}$ converges to some point $\varrho \in X$, that is $\lim _{n \rightarrow \infty} x_{n}=\varrho$, and $d(\varrho, \varrho)=0$. As $d(\varrho, \varrho)=\lim _{n \rightarrow \infty} d\left(x_{n}, \varrho\right)=\lim _{n, m \rightarrow \infty} d\left(x_{n}, x_{m}\right)=0$.

Case-I: If $T$ is continuous. Then we have,

$$
\varrho=\lim _{n \rightarrow \infty} x_{n+1}=\lim _{n \rightarrow \infty} T x_{n}=T\left(\lim _{n \rightarrow \infty} x_{n}\right)=T \varrho
$$

and so, $\varrho$ is a fixed point of $T$.

Case-II: If $F$ is continuous. In this case, we will prove that $\varrho=T \varrho$. Let us assume that $\varrho \neq T \varrho$.

In this case, there exists an $n_{0} \in \mathbb{N}$ and a subsequence $\left\{x_{n_{e}}\right\}$ of $\left\{x_{n}\right\}$ such that $d\left(T x_{n_{e}}, T \varrho\right)>0$ for all $n_{e} \geq n_{0}$.

Otherwise, if not, there exist $n_{1} \in \mathbb{N}$ such that $x_{n}=T \varrho$ for all $n \geq n_{1}$, which yields that $x_{n} \rightarrow T \varrho$. This is a contradiction, since $\varrho \neq T \varrho$. Being $d\left(T x_{n_{e}}, T \varrho\right)>0$ for all $n_{e} \geq n_{0}$, then from hypothesis, we have,

$$
\begin{aligned}
\tau+F\left(d\left(x_{n_{e+1}}, T \varrho\right)\right) & =\tau+F\left(d\left(T x_{n_{e+1}}, T \varrho\right)\right) \\
& \leq F\left(\mathcal{A}\left(x_{n_{e}}, \varrho\right)\right)
\end{aligned}
$$

where,

$$
\begin{gathered}
\mathcal{A}\left(x_{n_{e}}, \varrho\right)=\max \left\{d\left(x_{n_{e}}, \varrho\right), d\left(x_{n_{e}}, T x_{n_{e}}\right), d(\varrho, T \varrho), \frac{d\left(x_{n_{e}}, T x_{n_{e}}\right)+d(\varrho, T \varrho)}{2},\right. \\
\frac{d(\varrho, T \varrho)\left[1+d\left(x_{n_{e}}, T x_{n_{e}}\right)\right]}{1+d\left(x_{n_{e}}, \varrho\right)}, \frac{d\left(x_{n_{e}}, T x_{n_{e}}\right) d(\varrho, T \varrho)}{1+d\left(x_{n_{e}}, \varrho\right)}, \\
\left.\frac{d^{2}\left(x_{n_{e}}, T x_{n_{e}}\right) d(\varrho, T \varrho)}{1+d^{2}\left(x_{n_{e}}, \varrho\right)}\right\}
\end{gathered}
$$

From (47),

$$
\begin{gathered}
\tau+F\left(d\left(x_{n_{e}+1}, T \varrho\right)\right)=\max \left\{d\left(x_{n_{e}}, \varrho\right), d\left(x_{n_{e}}, x_{n_{e}+1}\right), d(\varrho, T \varrho), \frac{d\left(x_{n_{e}}, x_{n_{e}+1}\right)+d(\varrho, T \varrho)}{2},\right. \\
\frac{d(\varrho, T \varrho)\left[1+d\left(x_{n_{e}}, x_{n_{e}+1}\right)\right]}{1+d\left(x_{n_{e}}, \varrho\right)}, \frac{d\left(x_{n_{e}}, x_{n_{e}+1}\right) d(\varrho, T \varrho)}{1+d\left(x_{n_{e}}, \varrho\right)}, \\
\left.\frac{d^{2}\left(x_{n_{e}}, x_{n_{e}+1}\right) d(\varrho, T \varrho)}{1+d^{2}\left(x_{n_{e}}, \varrho\right)}\right\}
\end{gathered}
$$


Taking the limit $n_{e} \rightarrow \infty$ and using the continuity of $F$, we get,

$$
\begin{aligned}
\tau+F(d(\varrho, T \varrho)) & \leq \max \left\{d(\varrho, \varrho), d(\varrho, T \varrho), \frac{d(\varrho, \varrho)+d(\varrho, T \varrho)}{2}\right\} \\
& \leq \max \{d(\varrho, \varrho), d(\varrho, T \varrho)\}
\end{aligned}
$$

Case-A: If $\max \{d(\varrho, \varrho), d(\varrho, T \varrho)\}=d(\varrho, T \varrho)$.

Then from (50), we get,

$$
\tau+F(d(\varrho, T \varrho)) \leq d(\varrho, T \varrho),
$$

which is a contradiction as $\tau>0$.

Case-B: If $\max \{d(\varrho, \varrho), d(\varrho, T \varrho)\}=d(\varrho, \varrho)$.

Then from (50), we get,

$$
\begin{aligned}
& \tau+F(d(\varrho, T \varrho)) \leq d(\varrho, \varrho), \\
& \Rightarrow \tau+F(d(\varrho, T \varrho)) \leq F(0),
\end{aligned}
$$

which is a contradiction.

Thus, in the both cases, we get the contradiction due to $d\left(x_{n_{e}+1}, T \varrho\right)>0$. Therefore, $x_{n_{e}+1}=T \varrho$, which implies $T x_{n_{e}}=T \varrho$. Thus, $T \varrho=\varrho$. This gives, $\varrho$ is a fixed point of $T$. To prove uniqueness, let $\varrho$ and $\varrho^{*}$ are two fixed points of $T$, i.e. $T \varrho=\varrho$ and $T \varrho^{*}=\varrho^{*}$.

Let us assume that $\varrho \neq \varrho^{*}$.

$$
\begin{gathered}
\Rightarrow d\left(\varrho, \varrho^{*}\right)>0 \\
\Rightarrow d\left(T \varrho, T \varrho^{*}\right)>0
\end{gathered}
$$

From hypothesis, we get,

$$
\tau+F\left(d\left(T \varrho, T \varrho^{*}\right)\right) \leq F\left(\mathcal{A}\left(\varrho, \varrho^{*}\right)\right)
$$

where,

$$
\begin{aligned}
\mathcal{A}\left(\varrho, \varrho^{*}\right)=\max \left\{d\left(\varrho, \varrho^{*}\right), d(\varrho, T \varrho), d\left(\varrho^{*}, T \varrho^{*}\right), \frac{d(\varrho, T \varrho)+d\left(\varrho^{*}, T \varrho^{*}\right)}{2}\right. & \frac{d\left(\varrho^{*}, T \varrho^{*}\right)[1+d(\varrho, T \varrho)]}{1+d\left(\varrho, \varrho^{*}\right)}, \frac{d(\varrho, T \varrho) d\left(\varrho^{*}, T \varrho^{*}\right)}{1+d\left(\varrho, \varrho^{*}\right)} \\
\left.\frac{d^{2}(\varrho, T \varrho) d\left(\varrho^{*}, T \varrho^{*}\right)}{1+d^{2}\left(\varrho, \varrho^{*}\right)}\right\} & \\
= & \max \left\{d\left(\varrho, \varrho^{*}\right), d(\varrho, \varrho), d\left(\varrho^{*}, \varrho^{*}\right), \frac{d(\varrho, \varrho)+d\left(\varrho^{*}, \varrho^{*}\right)}{2},\right. \\
\frac{d\left(\varrho^{*}, \varrho^{*}\right)[1+d(\varrho, \varrho)]}{1+d\left(\varrho, \varrho^{*}\right)}, \frac{d(\varrho, \varrho) d\left(\varrho^{*}, \varrho^{*}\right)}{1+d\left(\varrho, \varrho^{*}\right)} & \left.\frac{d^{2}(\varrho, \varrho) d\left(\varrho^{*}, \varrho^{*}\right)}{1+d^{2}\left(\varrho, \varrho^{*}\right)}\right\} \\
= & \max \left\{d\left(\varrho, \varrho^{*}\right)\right\} \\
= & d\left(\varrho, \varrho^{*}\right)
\end{aligned}
$$


From hypothesis, we get,

$$
\begin{aligned}
& \tau+F\left(d\left(T \varrho, T \varrho^{*}\right)\right) \leq F\left(d\left(\varrho, \varrho^{*}\right)\right) \\
& \Rightarrow \tau+F\left(d\left(\varrho, \varrho^{*}\right)\right) \leq F\left(d\left(\varrho, \varrho^{*}\right)\right),
\end{aligned}
$$

which is a contradiction. Hence $\varrho=\varrho^{*}$, this completes the proof of the theorem.

Corollary 3. Let $(X, d)$ be a dislocated metric space such that $d$ is continuous functional. If $T$ is a Weak-generalized F-contraction, i.e., there exist $F$ along with $C_{1}, C_{2}, C_{3}$ and $\tau>0$ such that $\forall x, y \in X$,

$$
d(T x, T y)>0 \Rightarrow \tau+F(d(T x, T y)) \leq F(\mathcal{O}(x, y))
$$

where

$$
\begin{array}{r}
\mathcal{O}(x, y)=\max \left\{d(x, y), d(x, T x), d(y, T y), \frac{d(x, T x)+d(y, T y)}{2},\right. \\
\frac{d(x, T x) d(y, T y)}{1+d(x, y)}, \frac{d(y, T y)[1+d(x, T x)]}{1+d(x, y)}, \\
\left.\frac{d^{2}(x, T x) d(y, T y)}{1+d^{2}(x, y)}\right\} .
\end{array}
$$

Then T has a unique fixed point.

\section{Application to Non-linear Integral Equation}

Now we study the existence of unique solutions for non-linear integral equations as an application using the Theorem 1.

Let us consider the non-linear integral equation:

$$
\Xi(i)=\int_{0}^{t} \Lambda(i, j) \Gamma(j, \Xi(j)) d j
$$

for all $i, j \in[0, T]$, where the unknown function $\Xi(i)$ takes real values.

Let $X=\mathbb{C}([0, T])$ be the space of all real continuous functions defined on $[0, T]$. We endow $X$ with the $d$-metric $d: X \times X \rightarrow \mathbb{R}$ by

$$
d(\Xi, \Theta)=\sup _{t \in[0, T]}(|\Xi(i)+\Theta(i)|)^{2}
$$

for all $\Xi, \Theta \in X$.

Clearly $(X, d, 2)$ is a complete $b$-dislocated metric space.

Let for all $j \in[0, T], \Gamma\left(j_{,}.\right)$be a decreasing function, that is:

$$
x, y \in \mathbb{R}, \quad x \geq y \Rightarrow \Gamma(j, x) \leq \Gamma(j, y)
$$

Define a mapping $T: X \rightarrow X$ by

$$
T \Xi(i)=\int_{0}^{i} \Lambda(i, j) \Gamma(j, \Xi(j)) d j ; \text { for all } i \in[0, T] .
$$

Furthermore, we assume the following conditions:

N1: $\quad \Lambda(i, j) \leq e^{\frac{j \tau}{2}}$ where $\tau>0$ and $j \in[0, T]$ 
N2: For all $j \in[0, T]$ and $\Xi(j), \Theta(j) \in X$

$$
\begin{gathered}
|\Gamma(j, \Xi(j))|+|\Gamma(j, \Theta(j))| \leq\left[\frac{3 \mathcal{A}_{1}}{2}(\Xi(j)+\Theta(j))^{2}+\mathcal{A}_{2}\left[(\Xi(j)+T \Xi(j))^{2}+(\Theta(j)+T \Theta(j))^{2}\right]\right. \\
+\mathcal{A}_{3}\left(\frac{(\Xi(j)+T \Xi(j))^{2}+(\Theta(j)+T \Theta(j))^{2}}{6}\right) \\
\left.+\mathcal{A}_{4}\left(\frac{(2 \Xi(j))^{2}+(2 \Theta(j))^{2}}{8}\right)\right]^{\frac{1}{2}} \frac{\tau}{2} e^{\frac{-\tau}{2}}
\end{gathered}
$$

where $\mathcal{A}_{1}, \mathcal{A}_{2}, \mathcal{A}_{3}, \mathcal{A}_{4} \geq 0$ and $\frac{3 \mathcal{A}_{1}}{2}+2\left(\mathcal{A}_{2}+\mathcal{A}_{3}+\mathcal{A}_{4}\right)<1$.

For $\Xi \in X$, we define a norm $\|\Xi\|_{\tau}=\sup _{i \in[0, T]}|\Xi(i)| e^{-\tau i}$. It is easy to check that $\|\cdot\|_{\tau}$ is equivalent to the supremum norm $\|$.$\| in X$, and $X$ is endowed with the $d$-metric $d$ defined by

$$
\begin{aligned}
d(\Xi, \Theta) & =\|\Xi+\Theta\|_{\tau} \\
& =\sup _{i \in[0, T]}\left\{(|\Xi(i)|+|\Theta(i)|)^{2} e^{-i \tau}\right\} ; \quad \text { for } \Xi, \Theta \in X .
\end{aligned}
$$

Then $(X, d)$ is a complete $b$-dislocated metric space.

Now we will prove the existence of unique solution of the non-linear integral Equation (53).

Theorem 3. Let $(X, d)$ be a $b$-dislocated metric space as defined above with assuming the above conditions N1 and N2. Then the non-linear integral Equation (53) have a unique solution.

Proof: Let $\Xi, \Theta \in X$ and $i \in[0, T]$ then consider, 


$$
\begin{aligned}
& {[|T \Xi(i)|+|T \Theta(i)|]^{2}=\left(\left|\int_{0}^{i} \Lambda(i, j) \Gamma(j, \Xi(j)) d j\right|+\left|\int_{0}^{i} \Lambda(i, j) \Gamma(j, \Theta(j)) d j\right|\right)^{2}} \\
& =\left(\int_{0}^{i} \Lambda(i, j)|\Gamma(j, \Xi(j))| d j+\int_{0}^{i} \Lambda(i, j)|\Gamma(j, \Theta(j))| d j\right)^{2} \\
& =\left(\int_{0}^{i} \Lambda(i, j)(|\Gamma(j, \Xi(j))|+|\Gamma(j, \Theta(j))|) d j\right)^{2} \\
& \leq\left(\int _ { 0 } ^ { i } \left[\frac{3 \mathcal{A}_{1}}{2}(\Xi(j)+\Theta(j))^{2}+\mathcal{A}_{2}\left[(\Xi(j)+T \Xi(j))^{2}+(\Theta(j)+T \Theta(j))^{2}\right]\right.\right. \\
& +\mathcal{A}_{3}\left(\frac{(\Xi(j)+T \Xi(j))^{2}+(\Theta(j)+T \Theta(j))^{2}}{6}\right) \\
& \left.\left.+\mathcal{A}_{4}\left(\frac{(2 \Xi(j))^{2}+(2 \Theta(j))^{2}}{8}\right)\right]^{\frac{1}{2}} \frac{\tau}{2} e^{\frac{-\tau}{2}} d j\right)^{2} \\
& \leq\left(\int _ { 0 } ^ { i } \Lambda ( i , j ) \left[\frac{3 \mathcal{A}_{1}}{2}(|\Xi(j)|+|\Theta(j)|)^{2}+\mathcal{A}_{2}\left[(|\Xi(j)|+|T \Xi(j)|)^{2}+(|\Theta(j)|+|T \Theta(j)|)^{2}\right]\right.\right. \\
& +\mathcal{A}_{3}\left(\frac{(|\Xi(j)|+|T \Xi(j)|)^{2}+(|\Theta(j)|+|T \Theta(j)|)^{2}}{6}\right) \\
& \left.\left.+\mathcal{A}_{4}\left(\frac{(2|\Xi(j)|+2|\Theta(j)|)^{2}}{8}\right)\right]^{\frac{1}{2}} \frac{\tau}{2} e^{\frac{-\tau}{2}} d j\right)^{2} \\
& \leq\left(\int _ { 0 } ^ { i } \Lambda ( i , j ) e ^ { j \tau } \left(e ^ { - j \tau } \left[\frac{3 \mathcal{A}_{1}}{2}(|\Xi(j)|+|\Theta(j)|)^{2}+\mathcal{A}_{2}\left[(|\Xi(j)|+|T \Xi(j)|)^{2}+(|\Theta(j)|+|T \Theta(j)|)^{2}\right]\right.\right.\right. \\
& +\mathcal{A}_{3}\left(\frac{(|\Xi(j)|+|T \Xi(j)|)^{2}+(|\Theta(j)|+|T \Theta(j)|)^{2}}{6}\right) \\
& \left.\left.\left.+\mathcal{A}_{4}\left(\frac{(2|\Xi(j)|+2|\Theta(j)|)^{2}}{8}\right)\right]^{\frac{1}{2}} \frac{\tau}{2} e^{\frac{-\tau}{2}} d j\right)\right)^{2} \\
& \leq\left(\int _ { 0 } ^ { i } \Lambda ( i , j ) e ^ { j \tau } \left[\frac{3 \mathcal{A}_{1}}{2} d(\Xi, \Theta)+\mathcal{A}_{2}[d(\Xi, T \Xi)+d(\Theta, T \Theta)]\right.\right. \\
& +\mathcal{A}_{3}\left(\frac{d(\Xi, T \Theta)+d(\Theta, T \Xi)}{6}\right) \\
& \left.\left.\left.+\mathcal{A}_{4}\left(\frac{d(\Xi, \Xi)+d(\Theta, \Theta)}{8}\right)\right]^{\frac{1}{2}} \frac{\tau}{2} e^{\frac{-\tau}{2}} d j\right)\right)^{2} \\
& \leq \frac{\tau^{2}}{4} e^{-\tau}\left(\int _ { 0 } ^ { i } \Lambda ( i , j ) \left[\frac{3 \mathcal{A}_{1}}{2} d(\Xi, \Theta)+\mathcal{A}_{2}[d(\Xi, T \Xi)+d(\Theta, T \Theta)]\right.\right. \\
& +\mathcal{A}_{3}\left(\frac{d(\Xi, T \Theta)+d(\Theta, T \Xi)}{6}\right) \\
& \left.\left.+\mathcal{A}_{4}\left(\frac{d(\Xi, \Xi)+d(\Theta, \Theta)}{8}\right)\right]^{\frac{1}{2}} d j\right)^{2} \\
& =\frac{\tau^{2}}{4} e^{-\tau}\left[\frac{3 \mathcal{A}_{1}}{2} d(\Xi, \Theta)+\mathcal{A}_{2}[d(\Xi, T \Xi)+d(\Theta, T \Theta)]\right. \\
& +\mathcal{A}_{3}\left(\frac{d(\Xi, T \Theta)+d(\Theta, T \Xi)}{6}\right) \\
& \left.+\mathcal{A}_{4}\left(\frac{d(\Xi, \Xi)+d(\Theta, \Theta)}{8}\right)\right]\left(\int_{0}^{i} \Lambda(i, j) d j\right)^{2}
\end{aligned}
$$




$$
\begin{aligned}
& {[|T \Xi(i)|+|T \Theta(i)|]^{2} \leq \frac{\tau^{2}}{4} e^{-\tau}\left[\frac{3 \mathcal{A}_{1}}{2} d(\Xi, \Theta)+\mathcal{A}_{2}[d(\Xi, T \Xi)+d(\Theta, T \Theta)]\right.} \\
& +\mathcal{A}_{3}\left(\frac{d(\Xi, T \Theta)+d(\Theta, T \Xi)}{6}\right) \\
& \left.+\mathcal{A}_{4}\left(\frac{d(\Xi, \Xi)+d(\Theta, \Theta)}{8}\right)\right]\left(\int_{0}^{i} e^{\frac{j \tau}{2}} d j\right)^{2} \\
& =\frac{\tau^{2}}{4} e^{-\tau}\left[\frac{3 \mathcal{A}_{1}}{2} d(\Xi, \Theta)+\mathcal{A}_{2}[d(\Xi, T \Xi)+d(\Theta, T \Theta)]\right. \\
& +\mathcal{A}_{3}\left(\frac{d(\Xi, T \Theta)+d(\Theta, T \Xi)}{6}\right) \\
& \left.+\mathcal{A}_{4}\left(\frac{d(\Xi, \Xi)+d(\Theta, \Theta)}{8}\right)\right]\left(\left(\frac{e^{\frac{j \tau}{2}}}{\frac{\tau}{2}}\right)_{0}^{i}\right)^{2} \\
& <\frac{\tau^{2}}{4} e^{-\tau}\left[\frac{3 \mathcal{A}_{1}}{2} d(\Xi, \Theta)+\mathcal{A}_{2}[d(\Xi, T \Xi)+d(\Theta, T \Theta)]\right. \\
& +\mathcal{A}_{3}\left(\frac{d(\Xi, T \Theta)+d(\Theta, T \Xi)}{6}\right) \\
& \left.+\mathcal{A}_{4}\left(\frac{d(\Xi, \Xi)+d(\Theta, \Theta)}{8}\right)\right]\left(e^{\frac{i \tau}{2}} \times \frac{2}{\tau}\right)^{2} \\
& =\frac{\tau^{2}}{4} e^{-\tau}\left[\frac{3 \mathcal{A}_{1}}{2} d(\Xi, \Theta)+\mathcal{A}_{2}[d(\Xi, T \Xi)+d(\Theta, T \Theta)]\right. \\
& +\mathcal{A}_{3}\left(\frac{d(\Xi, T \Theta)+d(\Theta, T \Xi)}{6}\right) \\
& \left.+\mathcal{A}_{4}\left(\frac{d(\Xi, \Xi)+d(\Theta, \Theta)}{8}\right)\right]\left(e^{i \tau} \frac{4}{\tau^{2}}\right) \\
& =e^{-\tau(1-i)}\left[\frac{3 \mathcal{A}_{1}}{2} d(\Xi, \Theta)+\mathcal{A}_{2}[d(\Xi, T \Xi)+d(\Theta, T \Theta)]\right. \\
& +\mathcal{A}_{3}\left(\frac{d(\Xi, T \Theta)+d(\Theta, T \Xi)}{6}\right) \\
& \left.+\mathcal{A}_{4}\left(\frac{d(\Xi, \Xi)+d(\Theta, \Theta)}{8}\right)\right]
\end{aligned}
$$

which implies,

$$
\begin{aligned}
& (|T \Xi(i)|+|T \Theta(i)|)^{2} \leq e^{-\tau(1-i)}\left[\frac{3 \mathcal{A}_{1}}{2} d(\Xi, \Theta)+\mathcal{A}_{2}[d(\Xi, T \Xi)+d(\Theta, T \Theta)]\right. \\
& +\mathcal{A}_{3}\left(\frac{d(\Xi, T \Theta)+d(\Theta, T \Xi)}{6}\right) \\
& \left.+\mathcal{A}_{4}\left(\frac{d(\Xi, \Xi)+d(\Theta, \Theta)}{8}\right)\right] \\
& \Rightarrow(|T \Xi(i)|+|T \Theta(i)|)^{2} e^{-i \tau} \leq e^{-\tau}\left[\frac{3 \mathcal{A}_{1}}{2} d(\Xi, \Theta)+\mathcal{A}_{2}[d(\Xi, T \Xi)+d(\Theta, T \Theta)]\right. \\
& +\mathcal{A}_{3}\left(\frac{d(\Xi, T \Theta)+d(\Theta, T \Xi)}{6}\right) \\
& \left.+\mathcal{A}_{4}\left(\frac{d(\Xi, \Xi)+d(\Theta, \Theta)}{8}\right)\right]
\end{aligned}
$$


this gives,

$$
\begin{aligned}
d(T \Xi, T \Theta) \leq e^{-\tau}\left[\frac{3 \mathcal{A}_{1}}{2} d(\Xi, \Theta)+\mathcal{A}_{2}[d(\Xi, T \Xi)+d(\Theta, T \Theta)]\right. \\
+\mathcal{A}_{3}\left(\frac{d(\Xi, T \Theta)+d(\Theta, T \Xi)}{6}\right) \\
\left.+\mathcal{A}_{4}\left(\frac{d(\Xi, \Xi)+d(\Theta, \Theta)}{8}\right)\right]
\end{aligned}
$$

Applying logarithms on both sides, we get,

$$
\begin{aligned}
\ln (d(T \Xi, T \Theta)) \leq \ln \left(e^{-\tau}[\right. & \frac{3 \mathcal{A}_{1}}{2} d(\Xi, \Theta)+\mathcal{A}_{2}[d(\Xi, T \Xi)+d(\Theta, T \Theta)] \\
+ & \mathcal{A}_{3}\left(\frac{d(\Xi, T \Theta)+d(\Theta, T \Xi)}{6}\right) \\
& \left.\left.+\mathcal{A}_{4}\left(\frac{d(\Xi, \Xi)+d(\Theta, \Theta)}{8}\right)\right]\right) \\
\leq \ln e^{-\tau}+\ln \left(\left[\frac{3 \mathcal{A}_{1}}{2} d(\Xi, \Theta)+\mathcal{A}_{2}[d(\Xi, T \Xi)+d(\Theta, T \Theta)]\right.\right. & \\
+ & \mathcal{A}_{3}\left(\frac{d(\Xi, T \Theta)+d(\Theta, T \Xi)}{6}\right) \\
& \left.\left.+\mathcal{A}_{4}\left(\frac{d(\Xi, \Xi)+d(\Theta, \Theta)}{8}\right)\right]\right) \\
( & {\left[\frac{3 \mathcal{A}_{1}}{2} d(\Xi, \Theta)+\mathcal{A}_{2}[d(\Xi, T \Xi)+d(\Theta, T \Theta)]\right.} \\
+ & \mathcal{A}_{3}\left(\frac{d(\Xi, T \Theta)+d(\Theta, T \Xi)}{6}\right) \\
+ & \left.\left.\mathcal{A}_{4}\left(\frac{d(\Xi, \Xi)+d(\Theta, \Theta)}{8}\right)\right]\right) \\
\tau+\ln (d(T \Xi, T \Theta)) \leq \ln ( & {\left[\frac{3 \mathcal{A}_{1}}{2} d(\Xi, \Theta)+\mathcal{A}_{2}[d(\Xi, T \Xi)+d(\Theta, T \Theta)]\right.} \\
+\mathcal{A}_{3}\left(\frac{d(\Xi, T \Theta)+d(\Theta, T \Xi)}{6}\right) & \left.\left.+\mathcal{A}_{4}\left(\frac{d(\Xi, \Xi)+d(\Theta, \Theta)}{8}\right)\right]\right)
\end{aligned}
$$

Define $F: \mathbb{R}^{+} \rightarrow \mathbb{R}$ by $F(\alpha)=\ln (\alpha), \quad \alpha>0$. Then from (61), we get

$$
\begin{aligned}
\tau+F(d(T \Xi, T \Theta)) \leq F\left(\left[\frac{3 \mathcal{A}_{1}}{2} d(\Xi, \Theta)+\mathcal{A}_{2}[d(\Xi, T \Xi)+d(\Theta, T \Theta)]\right.\right. \\
+\mathcal{A}_{3}\left(\frac{d(\Xi, T \Theta)+d(\Theta, T \Xi)}{6}\right) \\
\left.\left.+\mathcal{A}_{4}\left(\frac{d(\Xi, \Xi)+d(\Theta, \Theta)}{8}\right)\right]\right)
\end{aligned}
$$


Thus, $T$ is an Extended F-contraction and satisfied all the conditions of the Theorem 1 . Thus, $T$ has a unique fixed point which is the unique solution of the non-linear integral equation.

\section{Application to Fractional Calculus}

In this section, we will start with the existence of solution for the non-linear fractional differential equation.

$$
C_{D^{\omega}}(x(\kappa))=\Lambda(\kappa, x(\kappa)), \quad(0<\kappa<1,1<\omega \leq 2)
$$

via the integral boundary conditions

$$
x(0)=0, x(1)=\int_{0}^{\xi} x(s) d s, \quad(0<\xi<1)
$$

where $C_{D^{\omega}}$ denotes the Caputo fractional derivative of order $\omega$ and $\Lambda:[0,1] \times \mathbb{R} \rightarrow \mathbb{R}$ is a continuous function. Let the space $X=\mathbb{C}([0,1], \mathbb{R})$ be the set of real continuous functions on $[0,1]$. We confer $X$ with the dislocated metric

$$
D_{\mathcal{M}(\infty)}(x, y)=\sup _{\kappa \in[0,1]}(|x(\kappa)|+|y(\kappa)|), \text { for all } x, y \in x
$$

Then $\left(X, D_{\mathcal{M}(\infty)}\right)$ is a complete dislocated metric space. It is very well known that, for a continuous function $\Theta:[0, \infty] \rightarrow \mathbb{R}$, the Caputo derivative of fractional order $\omega$ is defined as:

$$
C_{D^{\omega}}(\Theta(\kappa))=\frac{1}{\Gamma(n-\omega)} \int_{0}^{\kappa}(\kappa-s)^{n-\omega-1} \Theta^{n}(s) d s ; n-1<\omega<n, n=[\omega]+1,
$$

where $[\omega]$ is represented as integer part of the real number $\omega$ and $\Gamma$ is a gamma function.

Now, we consider the following conditions:

$\mathcal{C}_{1}:$ For $u, v \in \mathbb{R}, \tau>0$ and $\mathcal{A}_{1}<\frac{1}{2}$

$$
(|\Lambda(\kappa, u)|+|\Lambda(\kappa, v)|) \leq e^{-\tau} 2 \mathcal{A}_{1} \frac{\Gamma(\omega+1)}{5}(|u|+|v|)
$$

$\mathcal{C}_{2}:$ Define $T: X \rightarrow X$ by

$$
\begin{aligned}
T x(\kappa)= & \frac{1}{\Gamma \omega} \int_{0}^{\kappa}(\kappa-s)^{\mathscr{\omega}-1} \Lambda(s, x(s)) d s \\
& -\frac{2 \kappa}{\left(2-\xi^{2}\right) \Gamma(\mathcal{\omega})} \int_{0}^{1}(1-s)^{\mathscr{\omega}-1} \Lambda(s, x(s)) d s \\
& +\frac{2 \kappa}{\left(2-\xi^{2}\right) \Gamma(\omega)} \int_{0}^{\xi}\left(\int_{0}^{s}(s-m)^{\mathscr{\omega}-1} \Lambda(\ell, x(\ell)) d \ell\right) d s ; \text { for } \kappa \in[0,1]
\end{aligned}
$$

Theorem 4. If $\mathcal{C}_{1} \& \mathcal{C}_{2}$ are satisfied then the problem (67) has at least one solution.

Proof. It is very well known that $x \in X$ is a solution of (67) if and only if $x \in X$ is a solution of the integral equation.

$$
\begin{aligned}
x(\kappa)= & \frac{1}{\Gamma(\omega)} \int_{0}^{\kappa}(\kappa-s)^{\mathscr{\omega}-1} \Lambda(s, x(s)) d s \\
& -\frac{2 \kappa}{\left(2-\xi^{2}\right) \Gamma(\omega)} \int_{0}^{1}(1-s)^{\mathscr{\omega}-1} \Lambda(s, \mathcal{\omega}(s)) d s \\
& +\frac{2 \kappa}{\left(2-\xi^{2}\right) \Gamma(\omega)} \int_{0}^{\xi}\left(\int_{0}^{s}(s-m)^{\mathcal{D}-1} \Lambda(\ell, x(\ell)) d \ell\right) d s ; \text { for } \kappa \in[0,1]
\end{aligned}
$$

Then, the problem (67) is equivalent to find $x^{\star} \in X$ which is a fixed point of $T$. 
Now, let $x, y \in X$ for all $t \in[0,1]$.

Consider,

$$
\begin{aligned}
& |T x(\kappa)|+|T y(\kappa)|=\mid \frac{1}{\Gamma(\mathcal{\omega})} \int_{0}^{\kappa}(\kappa-s)^{\mathscr{\omega}-1} \Lambda(s, x(s)) d s
\end{aligned}
$$

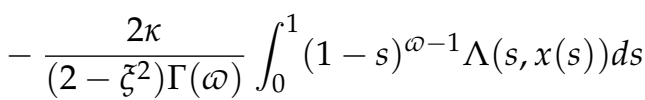

$$
\begin{aligned}
& +\frac{2 \kappa}{\left(2-\xi^{2}\right) \Gamma(\mathscr{\omega})} \int_{0}^{\xi}\left(\int_{0}^{s}(s-m)^{\mathscr{\omega}-1} \Lambda(\ell, x(\ell)) d \ell\right) d s \mid \\
& +\mid \frac{1}{\Gamma(\mathscr{\omega})} \int_{0}^{\kappa}(\kappa-s)^{\mathscr{\omega}-1} \Lambda(s, y(s)) d s
\end{aligned}
$$

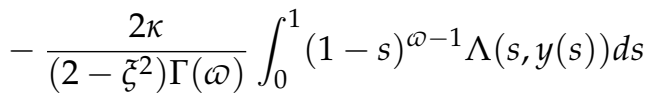

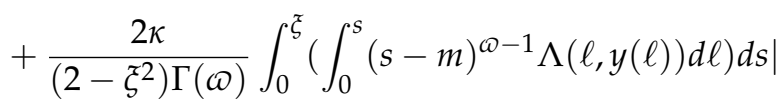

$$
\begin{aligned}
& =\mid \frac{1}{\Gamma(\mathscr{\omega})} \int_{0}^{\kappa}(\kappa-s)^{\mathscr{\omega}-1}[\Lambda(s, x(s))+\Lambda(s, y(s))] d s \\
& -\frac{2 \kappa}{\left(2-\xi^{2}\right) \Gamma(\mathscr{\omega})} \int_{0}^{1}(1-s)^{\mathscr{\omega}-1}[\Lambda(s, x(s)+\Lambda(s, y(s))] d s \\
& +\frac{2 \kappa}{\left(2-\xi^{2}\right) \Gamma(\mathscr{\omega})} \int_{0}^{\xi}\left(\int_{0}^{s}(s-m)^{\mathscr{D}-1}[\Lambda(\ell, x(\ell))+\Lambda(\ell, y(\ell))] d \ell\right) d s \mid \\
& \leq \frac{1}{\Gamma(\mathscr{\omega})} \int_{0}^{\kappa}(\kappa-s)^{\mathscr{\omega}-1}[|\Lambda(s, x(s))+\Lambda(s, y(s))|] d s \\
& -\frac{2 \kappa}{\left(2-\xi^{2}\right) \Gamma(\mathscr{\omega})} \int_{0}^{1}(1-s)^{\mathscr{\omega}-1}[\mid \Lambda(s, x(s)+\Lambda(s, y(s)) \mid] d s \\
& +\frac{2 \kappa}{\left(2-\xi^{2}\right) \Gamma(\omega)} \int_{0}^{\tilde{\zeta}}\left(\int_{0}^{s}(s-m)^{\omega-1}[|\Lambda(\ell, x(\ell))+\Lambda(\ell, y(\ell))|] d \ell\right) d s \\
& \leq \frac{1}{\Gamma(\mathscr{\omega})} \int_{0}^{\kappa}(\kappa-s)^{\mathscr{\omega}-1}[|\Lambda(s, x(s))|+|\Lambda(s, y(s))|] d s \\
& -\frac{2 \kappa}{\left(2-\xi^{2}\right) \Gamma(\mathscr{\omega})} \int_{0}^{1}(1-s)^{\mathscr{D}-1}[\mid \Lambda(s, x(s)|+| \Lambda(s, y(s)) \mid] d s \\
& +\frac{2 \kappa}{\left(2-\xi^{2}\right) \Gamma(\omega)} \int_{0}^{\xi}\left(\int_{0}^{s}(s-m)^{\omega-1}[|\Lambda(\ell, x(\ell))|+|\Lambda(\ell, y(\ell))|] d \ell\right) d s \\
& \leq \frac{\Gamma \omega+1}{5}[|x(s)|+|y(s)|] e^{-\tau} \sup \left\{\frac{1}{\Gamma \omega} \int_{0}^{\kappa}(\kappa-s)^{\omega-1} d s\right. \\
& \left.-\frac{2 \kappa}{\left(2-\xi^{2}\right) \Gamma(\mathscr{\omega})} \int_{0}^{1}(1-s)^{\mathscr{\omega}-1} d s+\frac{2 \kappa}{\left(2-\xi^{2}\right) \Gamma(\mathscr{\omega})} \int_{0}^{\xi}\left(\int_{0}^{s}(s-m)^{\mathscr{\omega}-1} d \ell\right) d s\right\} \\
& \leq e^{-\tau} 2 \mathcal{A}_{1}|x(s)|+|y(s)|
\end{aligned}
$$

By taking supremum on both sides, we get,

$$
D_{\mathcal{M}(\infty)}(T x, T y) \leq e^{-\tau} 2 \mathcal{A}_{1} D_{\mathcal{M}(\infty)}(x, y) .
$$

Taking logarithms on both sides, $\ln \left(D_{\mathcal{M}(\infty)}(T x, T y)\right) \leq-\tau+\ln \left(2 \mathcal{A}_{1} D_{\mathcal{M}(\infty)}(x, y)\right)$, which implies, $F\left(D_{\mathcal{M}(\infty)}(T x, T y)\right)+\tau \leq F\left(2 \mathcal{A}_{1} D_{\mathcal{M}(\infty)}(x, y)\right)$.

By taking $F(\alpha)=\ln \alpha$ for $\alpha>0$ and $\mathcal{A}_{1}<\frac{1}{2}, \mathcal{A}_{2}, \mathcal{A}_{3}, \mathcal{A}_{4}=0$, all the conditions of Corollary 2 satisfied. Hence $T$ has a unique fixed point. i.e., $T x^{\star}=x^{\star}$. Hence $x^{\star}$ is a solution of (67).

Author Contributions: All authors contributed equally and significantly in writing this article. All authors read and approved the final manuscript. 
Funding: This research received no external funding.

Acknowledgments: The authors are grateful to the reviewers for their careful reviews and useful comments. The authors would like to extend their sincere appreciation to the Deanship of Scientific Research at King Saud University for funding this group No. RG-1440-025.

Conflicts of Interest: The authors declare that they have no conflicts of interest.

Data Availability: No data were used to support this study.

\section{References}

1. Wardowski, D. Fixed point theory of a new type of contractive mappings in complete metric spaces. Fixed Point Theory Appl. 2012, 94. [CrossRef]

2. Secelean, N.A. Iterated function systems consisting of F-contractions. Fixed Point Theory Appl. 2013, 277. [CrossRef]

3. Piri, H.; Kumam, P. Some fixed point theorems concerning F-contraction in complete metric spaces. Fixed Point Theory Appl. 2014, 2014, 210. [CrossRef]

4. Karapınar, E.; Kutbi, M.A.; Piri, H.; O’Regan, D. Fixed points of conditionally F-contractions in complete metric-like spaces. Fixed Point Theory Appl. 2015, 2015, 126. [CrossRef]

5. Wardowski, D.; Van Dung, N. Fixed points of $\mathrm{f}$-weak contractions on complete metric spaces. Demonstr. Math. 2014, 1, 146-155. [CrossRef]

6. Minak, G.; Halvaci, A.; Altun, I. Ciric type generalized F-contractions on complete metric spaces and fixed point results. Filomat 2014, 28, 1143-1151. [CrossRef]

7. Vetro, F. F-contractions of Hardy-Rogers type and application to multistage decision processes. Nonlinear Anal. Model. Control 2016, 21, 531-546.

8. Wardowski, D. Solving existence problems via F-contractions. Proc. Am. Math. Soc. 2018, 146, 1585-1598. [CrossRef]

9. Lukacs, A.; Kajanto, S. Fixed point theorems for various types of F-ontractions in complete b-metric spaces. Fixed Point Theory Appl. 2018, 19, 321-334. [CrossRef]

10. Rasham, T.; Shoaib, A.; Hussain, N.; Arshad, M.; Khan, S.U. Common fixed point results for new Ciric-type rational multivalued F-contraction with an application. Fixed Point Theory Appl. 2018, 20, 45. [CrossRef]

11. Kumari, P.S.; Panthi, D. Cyclic compatible contraction and related fixed point theorems. Fixed Point Theory Appl. 2016, 2016, 28. [CrossRef]

12. Dung, N.V.; Hang, V.T.L. A fixed point theorem for generalized F-contractions on complete metric spaces. Vietnam J. Math. 2015, 43, 743-753. [CrossRef]

13. Kumari, P.S.; Panthi, D. Connecting various types of cyclic contractions and contractive self-mappings with Hardy-Rogers self-mappings. Fixed Point Theory Appl. 2016, 2016, 15. [CrossRef]

14. Karapınar, E.; Kumari, P.S.; Lateef, D. A New Approach to the Solution of the Fredholm Integral Equation via a Fixed Point on Extended b-Metric Spaces. Symmetry 2018, 10, 512. [CrossRef]

15. Kumari, P.S.; Zoto, K.; Panthi, D. d-Neighborhood system and generalized F-contraction in dislocated metric space. SpringerPlus 2015, 4, 368. [CrossRef] [PubMed]

16. Matthews, S.G. Metric Domains for Completeness. Ph.D. Thesis, University of Warwick, Warwick, UK, 1985.

17. Hitzler, P.; Seda, A.K. Seda, Dislocated Topologies. J. Electr. Eng. 2000, 51, 3-7.

18. Alghamdi, M.A.; Hussain, N.; Salimi, P. Fixed point and coupled fixed point theorems on b-metric-like spaces. J. Inequal. Appl. 2013, 2013, 402. [CrossRef]

19. Chen, C.; Dong, J.; Zhu, C. Some fixed point theorems in b-metric-like spaces. Fixed Point Theory Appl. 2015, 2015, 122. [CrossRef]

20. Hussain, N.; Roshan, J.R.; Parvaneh, V.; Kadelburg, Z. Fixed points of contractive mappings in b-metric-like spaces. Sci. World J. 2014, 2014, 471827. [CrossRef]

21. Zoto, K.; Rhoades, B.E.; Radenović, S. Some generalizations for $(\alpha-\psi, \phi)$-contractions in b-metric-like spaces and an application. Fixed Point Theory Appl. 2017, 2017, 26. [CrossRef]

22. Aydi, H.; Felhi, A.; Sahmim, S. On common fixed points for $\alpha \psi$-contractions and generalized cyclic contractions in b-metric-like spaces and consequences. J. Nonlinear Sci. Appl. 2016, 9, 2492-2510. [CrossRef] 
23. Aydi, H.; Felhi, A.; Sahmim, S. Common fixed points via implicit contractions on b-metric-like spaces. J. Nonlinear Sci. Appl. 2017, 10, 1524-1537. [CrossRef]

24. Klin-eam, C.; Suanoom, C. Dislocated quasi-b-metric spaces and fixed point theorems for cyclic contractions. Fixed Point Theory Appl. 2015, 2015, 74. [CrossRef]

25. Hitzler, P. Generalized Metrics and Topology in Logic Programming Semantics. Ph.D. Thesis, National University of Ireland, University College, Cork, Ireland, 2001.

26. Karapınar, E. A Short Survey on Dislocated Metric Spaces via Fixed-Point Theory. In Advances in Nonlinear Analysis via the Concept of Measure of Noncompactness; Springer: Singapore, 2017; pp. 457-483.

(c) 2018 by the authors. Licensee MDPI, Basel, Switzerland. This article is an open access article distributed under the terms and conditions of the Creative Commons Attribution (CC BY) license (http://creativecommons.org/licenses/by/4.0/). 